\title{
Generator of Ultrashort Optical Pulses for Time Division Multiplexing
}

\author{
Jaume Dellunde, C. R. Mirasso, M. C. Torrent, and Jose M. Sancho
}

\begin{abstract}
The performance of a device based on modified injection-locking techniques is studied by means of numerical simulations. The device incorporates master and slave configurations, each one with a DFB laser and an electroabsortion modulator (EAM). This arrangement allows the generation of high peak power, narrow optical pulses according to a periodic or pseudorandom bit stream provided by a current signal generator. The device is able to considerably increase the modulation bandwidth of free-running gain-switched semiconductor lasers using multiplexing in the time domain. Opportunities for integration in small packages or single chips are discussed.
\end{abstract}

Index Terms - Distributed feedback lasers, electrooptic materials/devices, integrated optoelectronics, monolithic integrated circuits.

\section{INTRODUCTION}

$\mathbf{F}$ IBER optic communication systems were developed to obtain large bandwidths in the transmission of coded information [1], [2]. The bit rate of typical laser sources is constrained by the limitations inherent in applying the pulse modulation to the drive current of the laser diode [3], [4]. When a single semiconductor laser is used as the optical source, the modulation frequency is limited by the characteristic relaxation oscillation frequency of the particular laser used, around a few $\mathrm{GHz}$ [5], [6]. When the modulation frequency is pushed beyond this limiting value, pattern effects arise and the amplitude of the emitted pulses decrease, leading to reduced eye openings and errors at the receiver [7]-[12].

Laser structures based on multiquantum wells have been developed to moderately increase the modulation bandwidth of single emitters [13]-[15]. Other methods for achieving better performance include modelocking to an external cavity [16], optoelectronic feedback [17] and $Q$-switching of multisection structures with a saturable absorber [18]. An important drawback of these techniques, however, is the periodic nature of the pulse train, and as a consequence they do not allow the generation of coded messages. Methods that allow more

Manuscript received September 22, 1997; revised May 18, 1998. The review of this paper was arranged by Editor P. K. Bhattacharya. This work was supported by Comisión Interministerial de Ciencia y Tecnología, Project PB96-0421.

J. Dellunde and J. M. Sancho are with the Departament d'Estructura i Constituents de la Matèria, Facultat de Física, Universitat de Barcelona, E08028 Barcelona, Spain (e-mail: dellunde@noise.ecm.ub.es).

C. R. Mirasso is with the Departament de Física, Universitat de les Illes Balears, E-07071 Palma de Mallorca, Spain.

M. C. Torrent is with the Departament de Física i Enginyeria Nuclear, EUETIT, Universitat Politècnica de Catalunya, E-08222 Terrassa, Spain.

Publisher Item Identifier S 0018-9383(98)07464-4. flexibility are self-seeding [19]-[21], weak optical feedback [22]-[25], and optical injection [26]-[28].

Optical injection from a narrowband tunable master source is one of the most promising techniques. It was first used to provide tunable, small linewidth operation in steady-state [29]-[31]. The physical mechanisms underlying this improved performance are frequency locking to the master source and suppression of relaxation oscillations. During gain-switched and current modulated operation, the optically injected semiconductor laser shows a reduced time jitter and a considerably lower frequency chirp than the free-running laser [28]-[34]. These effects are beneficial and, in fact, several experimental setups have successfully implemented injection-locked devices in the above described way [26], [27], [35]. Further progress in the last years has come mainly from new designs of the single laser structures, while the injection-locking scheme remains the same.

Due to the moderate successes obtained working with single sources, alternative methods have been envisaged to overcome bandwidth limitations, including mainly multiplexing in the time or frequency domains [2], [4], [36]. Communication systems have already been implemented using wavelength division multiplexing, but this technique imposes severe linewidth requirements on the single emitters, and the manufacture of wavelength couplers, frequency converters, routers, demultiplexers and other elements performing specific tasks at high bit rates. Although using time division multiplexing the attainable transmission rate is not so high, the required technology to implement such links is less sophisticated and more robust.

Although time division multiplexing systems are forced to lower transmission rates, the technology needed to implement such links is less sophisticated, and the links seem to be more robust. Multiplexing in the time domain requires pseudorandom output of narrow, low jitter, high optical pulses from independent sources. In this way, several pulses can be combined in a single time slot and then demultiplexed at the receiver after transmission in a single-mode fiber.

The standard injection-locking technique assumes a stabilized master oscillator [29]-[31], [33], [34]. Efforts have been made to thermally stabilize the master output including electronic feedback loops. The turn-on process of the slave laser is thus triggered by the external seed supplied by the master source, instead of spontaneous emission events present in the active region of the slave [32]. The external seeding process forces an early turn on, reducing the time jitter but also leading to broader output pulses with a lower power peak, because the turn on occurs when the net gain is low [33], [34]. 


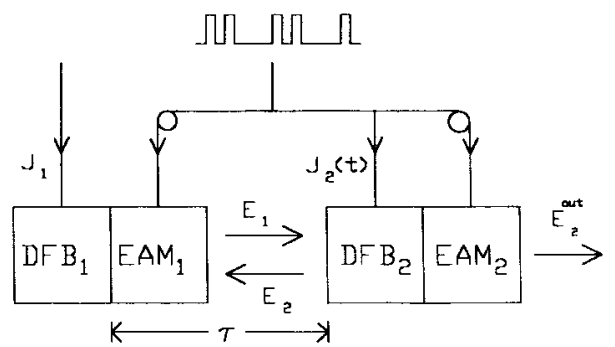

Fig. 1. Schematical sketch of the proposed device.

This drawback of the standard injection-locking technique puts limitations on the generation of optical pulses for multiplexing purposes in the time domain.

A modified scheme based on optical injection from a modulated laser diode was also proposed [37]. The master laser was gain-switched according to a pseudorandom bit stream and narrow, low jitter, low chirp, high peak power pulsed output was demonstrated by means of numerical simulations. This new effect was the result of optical injection at selected times after the gain-switch of the slave, so that the external seed was only supplied when the net gain was at its maximum. However, several limitations appeared soon when considering its practical implementation. The external seed was not at all stabilized, showing the characteristic time jitter, frequency chirp and peak power dispersion typical of gain-switched operation [38], [39]. The arrangement required the use of an optical fiber loop and a Fabry-Pérot filter, which would prevent the miniaturization in an integrated device. Possibly the main drawback, however, was the appearance in the final output of secondary pulses before and after the main pulse, with a typical width comparable to that of the main pulse. These secondary pulses would interact with adjacent pulses leading to errors in the multiplexed output.

In this work we present a device based on optical injection, amenable to integration in a package, to be used as a single emitter of coded output for multiplexing purposes in the time domain.

\section{DEVICE DESCRIPTION}

The proposed device is composed of several optoelectronic devices combined in a particular way to get the desired performance. The device is sketched in Fig. 1. External inputs to the device are dc currents for biasing and modulated current from a signal generator. Section II-A descibes the optical arrangement, and the required driving circuitry is detailed in Section II-B.

\section{A. Optical Arrangement}

A single-mode semiconductor laser $\left(\mathrm{DFB}_{1}\right)$ is used as the master oscillator. The continuous wave output of $\mathrm{DFB}_{1}$ is supplied to a fast-response external modulator $\left(\mathrm{EAM}_{1}\right)$. Optionally, for miniaturization purposes, the external modulator can be monolithically integrated with the laser. In commercially available devices, EAM EA $_{1}$ is ually an electroabsorption modulator. The master configuration is formed by the master oscillator $\mathrm{DFB}_{1}$ and the external modulator $\mathrm{EAM}_{1}$.
The light beam emerging from the master configuration is then supplied to the active region of a second (preferably but not necessarily single-mode) semiconductor laser $\left(\mathrm{DFB}_{2}\right)$, working at the same wavelength as $\mathrm{DFB}_{1}$. The master output is injected to optically match the dominant mode of $\mathrm{DFB}_{2}$. The output beam from $\mathrm{DFB}_{2}$ is modified by means of another modulator $\left(\mathrm{EAM}_{2}\right)$ with a similar performance to $\mathrm{EAM}_{1}$. The slave configuration is formed by the semiconductor laser $\mathrm{DFB}_{2}$ and the modulator $\mathrm{EAM}_{2}$. The slave configuration is not typically envisioned in the form of a closed package as the master configuration, because it must allow for the injection of an external optical beam. The light emerging from $\mathrm{EAM}_{2}$ is finally considered as the output beam.

Alternatively, and more appropriate for integration purposes, the lasers $\mathrm{DFB}_{1}$ and $\mathrm{DFB}_{2}$ and the modulators $\mathrm{EAM}_{1}$ and $\mathrm{EAM}_{2}$ can be grown on the same substrate avoiding the use of collimating lenses. In usual injection-locking arrangements, an optical isolator must be placed connecting the master and slave configurations. This optical isolator is included in order to suppress any influence of the output beam on the $\mathrm{CW}$ operation of the master laser. In this way, control of both output power and frequency tuning can be achieved. Our device does not need such an optical isolator, as long as a careful adjustment of the distance between $\mathrm{EAM}_{1}$ and $\mathrm{DFB}_{2}$ is provided. This point will be clarified in Section IV.

\section{B. Driving Circuitry}

A dc current $J_{1}$ is supplied to $\mathrm{DFB}_{1}$ to drive the master oscillator well above its threshold value and thus provide stabilized output with a narrow linewidth. Further power or frequency stabilization is achieved by means of thermal stabilization. The dc current across $\mathrm{DFB}_{1}$ must be chosen to match the emission frequency of $\mathrm{DFB}_{2}$. The slave laser $\mathrm{DFB}_{2}$ is dc biased below its threshold value. A bit stream is superimposed to that dc current by means of a signal generator independent of the proposed device. The signal generator provides pulse coded information by means of 50 ps rectangular current pulses with 15 ps rise and fall times, in a return to zero (RZ) format. The modulation period is set at 200 ps. $\mathrm{DFB}_{2}$ is thus gain-switched in the standard way. The same modulated current is supplied to the external modulators $\mathrm{EAM}_{1}$ and $\mathrm{EAM}_{2}$ with appropiate delays. These delays caused by different lengths in the high frequency delay lines must be carefully selected. The delay time of $\mathrm{EAM}_{1}$ is chosen according to the performance of the laser $\mathrm{DFB}_{2}$ free of optical injection. The choice of the delay time of $\mathrm{EAM}_{2}$, by contrast, should be made after the mean width of the generated pulses is known. All these tricky adjustments can be avoided by inserting timing control circuits for externally varying the required modulator delays. Losses in the delay lines must match the required voltages of the external modulators.

\section{MODELING}

The performance of the proposed device is tested by numerical simulation of the several independent devices of the configuration. The single mode operation of the two lasers $\mathrm{DFB}_{1}$ and $\mathrm{DFB}_{2}$ is modeled by means of modified rate 
TABLE I

Parameters in the Modified Rate Equations

\begin{tabular}{ccc}
\hline Parameter & Meaning & Value \\
\hline$\omega_{i}$ & emission angular frequency & $1.2210^{15} \mathrm{~s}^{-1}$ \\
$\alpha_{i}$ & linewidth enhancement factor & 5 \\
$g_{i}$ & differential gain & $1.510^{4} \mathrm{~s}^{-1}$ \\
$s_{i}$ & gain compression factor & $410^{-7}$ \\
$\gamma_{i}$ & inverse photon lifetime & $510^{11} \mathrm{~s}^{-1}$ \\
$N_{0 i}$ & transparency value for the carrier number & $1.510^{8}$ \\
$\beta_{i}$ & spontaneous emission rate & $0.510^{4} \mathrm{~s}^{-1}$ \\
$\gamma_{e i}$ & inverse carrier lifetime & $510^{8} \mathrm{~s}^{-1}$ \\
$e J_{i}^{t h}$ & threshold current & $14.7 \mathrm{~mA}$ \\
$e J_{i}^{b i a s}$ & bias current & $14.4 \mathrm{~mA}$ \\
$e J_{i}^{\text {max }}$ & injection current peak & $51.5 \mathrm{~mA}$ \\
$k_{i}^{\prime}$ & injection coupling & $0.15 \mathrm{ps}$ \\
$\eta_{e x t}^{2}$ & coupling losses & 1 \\
$\tau$ & injection delay & $30 \mathrm{ps}$ \\
$a_{0}$ & modulator low-loss value at the top of a pulse & 0.02 \\
$a_{1}$ & modulator high-loss value at the bottom of a pulse & 30 \\
\hline
\end{tabular}

equations, widely used to describe the behavior of free-running lasers [40], lasers exposed to conventional [24] and phaseconjugate [41] optical feedback and optically injected [27] or self-seeded [42] oscillators. The modified rate equations for the amplitude of the optical fields and the carrier number inside the laser cavities are

$$
\begin{aligned}
\frac{d E_{i}}{d t}= & \frac{1+i \alpha_{i}}{2}\left[\frac{g_{i}\left(N_{i}(t)-N_{0 i}\right)}{1+s_{i}\left|E_{i}(t)\right|^{2}}-\gamma_{i}\right] \\
& \cdot E_{i}(t)+k_{i} E_{j}^{\prime}(t) e^{i\left(\omega_{j}-\omega_{i}\right) t} \\
& +\sqrt{2 \beta_{i} N_{i}(t)} \xi_{i}(t) \\
\frac{d N_{i}}{d t}= & J_{i}(t)-\gamma_{e i} N_{i}(t) \\
& -\frac{g_{i}\left(N_{i}(t)-N_{0 i}\right)}{1+s_{i}\left|E_{i}(t)\right|^{2}}\left|E_{i}(t)\right|^{2}
\end{aligned}
$$

valid for $i=1, j=2$, and $i=2, j=1$. The free-running operation of the lasers is perturbed by the injection terms $k_{i} E_{j}^{\prime}(t) e^{i\left(\omega_{j}-\omega_{i}\right) t} . k_{i}$ is related to the injection coupling parameter $k_{i}^{\prime}$, which depends on which particular laser structure is used, through

$$
k_{i}=k_{i}^{\prime} \eta_{\mathrm{ext}}^{i}
$$

and accounts for the coupling between the injected and the intracavity fields, suitably normalized. Power losses arising from mode matchings and other effects different to the losses introduced by the laser facet are considered. The parameter $\eta_{\text {ext }}^{i}$, lower than one, accounts for all these additional losses. The $E_{j}^{\prime}(t)$ field is the incoming beam to the laser $i$ arising from the laser $j$ at a time $t-\tau$. The time $\tau$ is the one required for the beams to travel trough the optical path linking $\mathrm{DFB}_{1}$ and $\mathrm{DFB}_{2}$. The input beams to the lasers are modified by the fast modulators $\mathrm{EAM}_{1}$ and $\mathrm{EAM}_{2}$, with field transfer function $h_{i}(t)$ (the same transfer function shifted in time is assumed for the two modulators). According to the above mentioned scheme, the incoming fields read

$$
\begin{aligned}
& E_{1}^{\prime}(t)=E_{1}(t-\tau) h_{1}(t-\tau) e^{-i \omega_{1} \tau} \\
& E_{2}^{\prime}(t)=E_{2}(t-\tau) e^{-\omega_{2} \tau} h_{1}(t) .
\end{aligned}
$$

The output beam from $\mathrm{DFB}_{2}$ is partially absorbed by $\mathrm{EAM}_{2}$, so that the total power obtained at the device output is evaluated as $E_{2}(t) h_{2}(t)$. The fast response transfer function of the modulators is related to $s_{i}(t)$, the normalized unbiased electrical modulator input through [43]

$$
h(t)=e^{-a_{0}-a_{1}\left(1-s_{i}(t)\right)}+0.02
$$

where $a_{0}$ is the low-loss value at the top of a pulse, while $a_{1}$ is the high-loss value at the bottom. A maximum extinction ratio of $34 \mathrm{~dB}$ [44] has been assumed for the two modulators. Spontaneous emission events are considered through complex Gaussian white noise terms $\xi_{i}(t)$ of zero mean and correlation

$$
\left\langle\xi_{i}\left(t_{1}\right) \xi_{j}^{*}\left(t_{2}\right)\right\rangle=2 \delta_{i j} \delta\left(t_{1}-t_{2}\right) .
$$


(a)

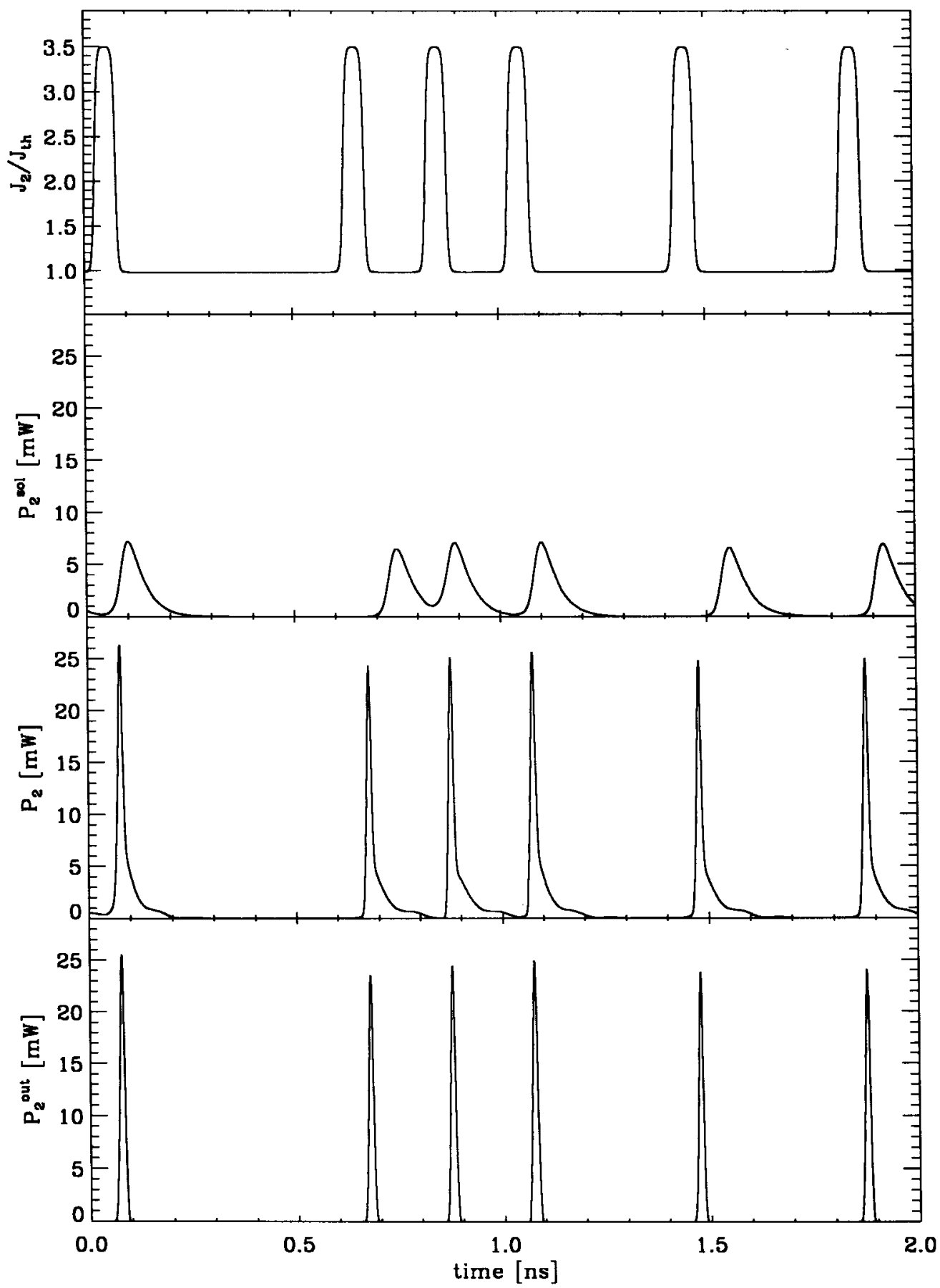

Fig. 2. Device response to a pseudorandom bit stream. (a) Input current, (b) solitary slave output, (c) optically injected slave output, and (d) total device output, are plotted. Here the counterpropagating coupling losses are set to $\eta_{\mathrm{ext}}^{1}=0.33$.

Although the parameters used, listed in Table I, are the same for the two lasers $\mathrm{DFB}_{1}$ and $\mathrm{DFB}_{2}$, the formalism employed remains valid when different independent choices of the oscillators are made to fit the desired performance. The injection current $J_{1}(t)=J_{1}$ is kept to a constant value above threshold, while $J_{2}(t)$ is the biased input from the signal generator. The frequency mismatch between the two lasers is set to zero due to the tuning provided to the master oscillator. In the above presented model, feedback effects due to optical reflections have been ignored. These effects should be included through Lang-Kobayashi [45] delayed feedback terms. In our numerical simulations we included such terms without any significant change in the results. This is due to the presence of direct optical injection terms, orders of magnitude more important than those arising from optical feedback.

The set of modified rate equations [(1), (2)] is numerically solved by means of a first-order Euler algorithm, with a time step of $0.01 \mathrm{ps}$, in order to obtain time traces of the output optical pulses. The time jitter of the generated pulses can be evaluated by averaging the turn-on time at a selected reference of the output power (usually at half the power peak) over many turn-on events. The chirped operation of the 
(a)

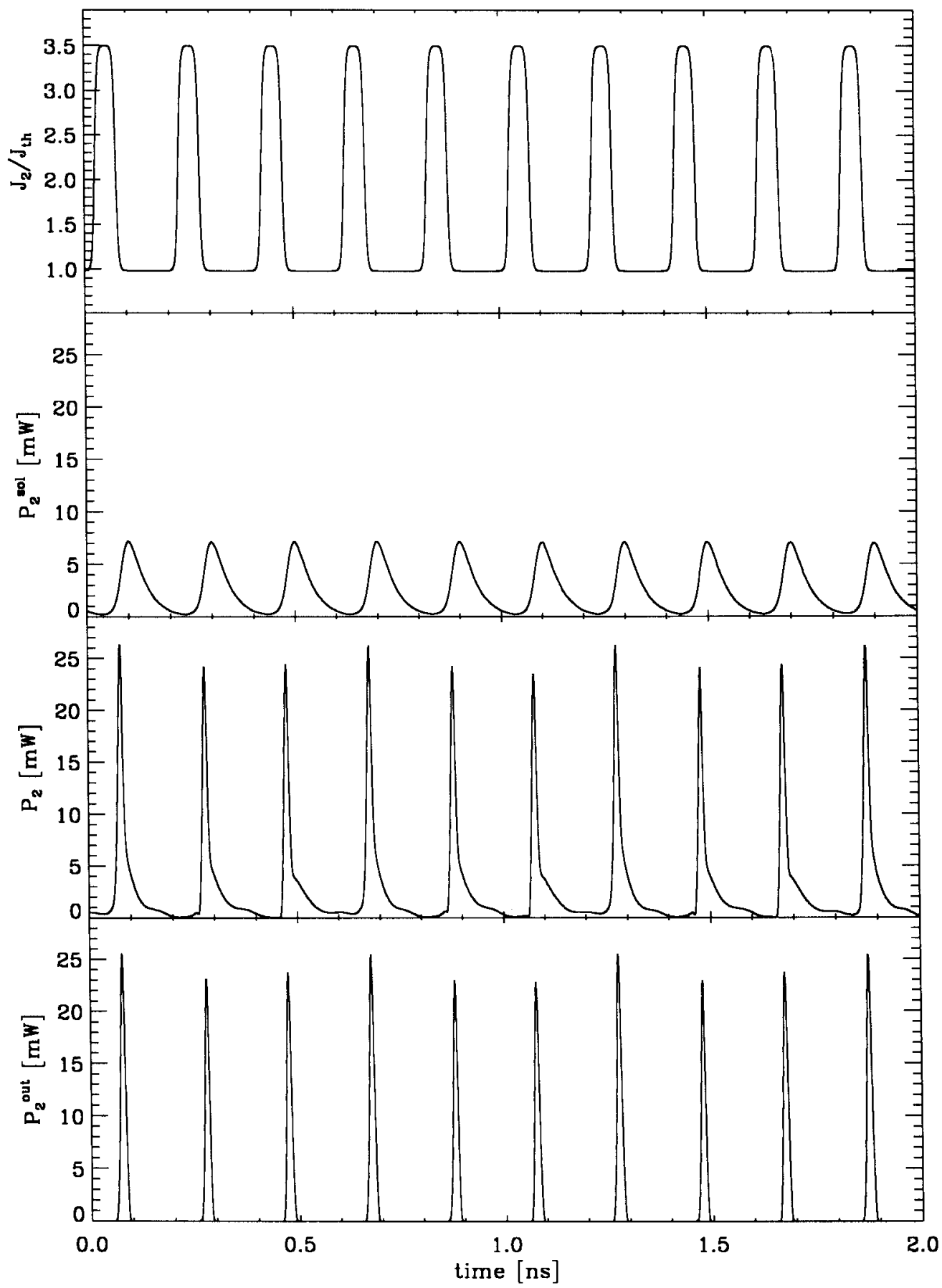

Fig. 3. The same as Fig. 2, but the device response to a periodic bit stream is considered.

generated output can be investigated by Fourier transforming the complex optical field inside a time slot. The performance of the proposed configuration can be optionally compared with that of the free-running slave laser $\mathrm{DFB}_{2}$, by setting the coupling losses $\eta_{\text {ext }}^{2}$ to zero and removing the external modulation supplied by $\mathrm{EAM}_{2}$.

\section{RESULTS}

According to the previous section, a pseudorandom bit stream at a modulation rate of $5 \mathrm{GHz}$ is supplied to the device. In Fig. 2(a), a 1001110101 current bit stream was used. The bias current was raised 3.5 times above its threshold value. Fig. 2(b) shows the optical response of the solitary laser $\mathrm{DFB}_{2}$ when no optical injection and optical modulators are considered. The peak power of the laser is around $7 \mathrm{~mW}$, and the pulse width is as large as to occupy the whole assigned time slot of 200 ps. Pattern effects [40] arise giving undesired pulse overlapping and distortion, leading to small eye openings. A higher optical pulse-peak is obtained by supplying optical injection from the master configuration to $\mathrm{DFB}_{2}$, as in Fig. 2(c) (coupling losses of $\eta_{\mathrm{ext}}^{1}=0.33$ are assumed for the counterpropagating wave). Optical pulses as high as $25 \mathrm{~mW}$ with small FWHM are achieved by this 
(a)

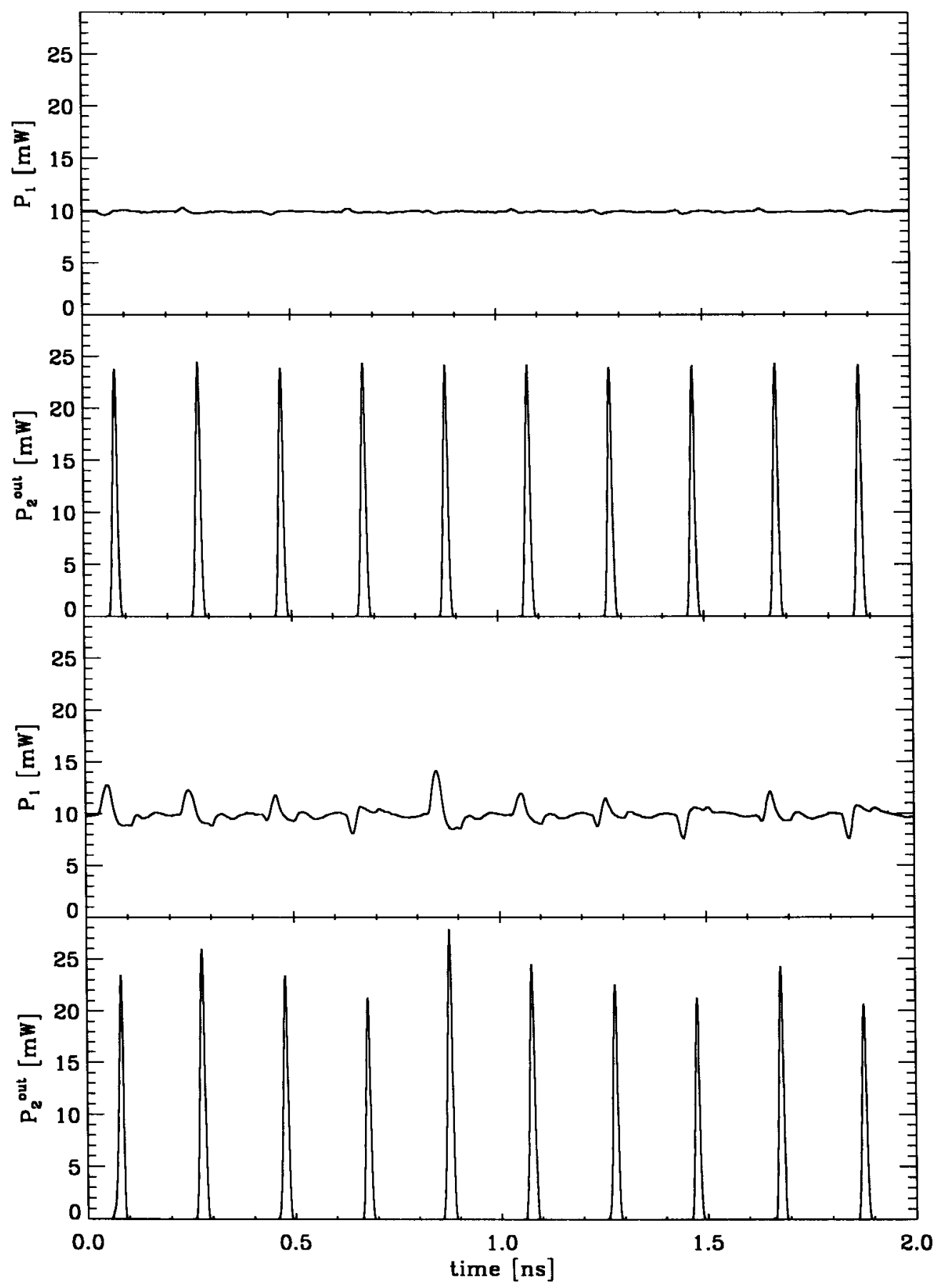

Fig. 4. Master output of $\mathrm{DFB}_{1}$ and total device output for two different values of the coupling losses: (a)-(b) $\eta_{\text {ext }}^{1}=0.13$ and (c)-(d) $\eta_{\text {ext }}^{1}=1$.

method. Nevertheless, we focus the attention of the reader on the large pulse widths at the bottom, which clearly prevents the implementation of multiplexing techniques in the time domain. This is why a second optical modulator $\mathrm{EAM}_{2}$ has been included in our device (see Fig. 2(d), where the final output power from the device is plotted). The modulator suppresses the pulse tails and is able to allocate the whole optical pulses of $25 \mathrm{~mW}$ in time slots narrower than $50 \mathrm{ps}$. In this way, up to four pulses can be multiplexed in the original time slot of $200 \mathrm{ps}$, leading to a potential $20 \mathrm{GHz}$ modulation frequency.

In the same direction as Fig. 2, the results concerning periodic instead of pseudorandom modulation are included in Fig. 3. In both cases the train of generated pulses was stable and the time jitter measured lower than 1 ps, so that the output pulses can be safely allocated in their assigned time slots. We attribute fluctuations of the peak-powers [see Figs. 2(d) and 3(d)] to the omission of an optical isolator which is present in typical injection-locking arrangements, giving rise to nonvanishing values of the coupling losses $\eta_{\text {ext }}^{1}$. The optical isolator is usually included to free the $\mathrm{CW}$ emission of the master laser from the influence of the slave. Our device has been designed without an optical isolator for low-cost and packaging purposes. There are two main reasons why it is important to remove the optical isolator used in 
(a)

(b)

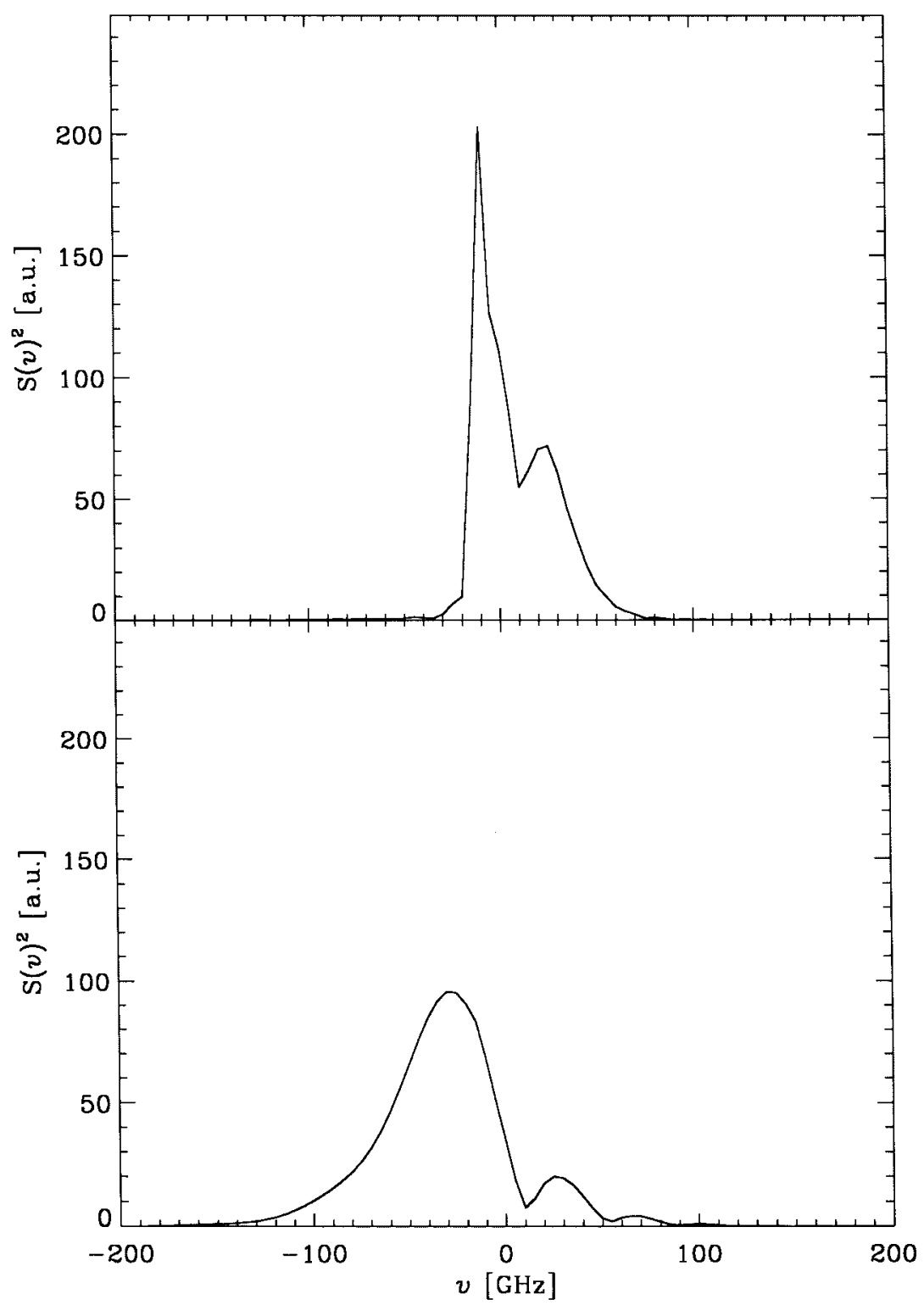

Fig. 5. The optical spectrum of (a) solitary gain-switched slave $\mathrm{DFB}_{2}$ is compared to the (b) optical spectrum obtained with the reported device.

standard injection-locking techniques. First of all, from an economical point of view, the optical isolator is the most expensive component of these systems, and its removal would significantly reduce the prize of the whole emitter. On the other hand, the optical isolator's size prevents the implementation of the emitter in a very small package and, as a consequence, its integrability. Whenever a large amount of laser emitters is required, it is necessary to remove the isolator. The main drawback due to the omission of the optical isolator is the need of including timing circuit controls or, alternatively, a careful positioning of the individual components. By selecting the distance between $\mathrm{EAM}_{1}$ and $\mathrm{DFB}_{2}$, the first modulator can be adjusted to receive light pulses from the slave when $\mathrm{EAM}_{1}$ is in the off state, thus preventing almost any external optical input to the master $\mathrm{DFB}_{1}$. The arrangement is similar to that reported in [46] designed to avoid optical feedback effects in DFB laser/electroabsorption modulator packages. Just to illustrate this point, Fig. 4 shows the output power of the dc biased master laser $\mathrm{DFB}_{1}$ and a train of optical pulses from the whole device. In Fig. 4(a) and (b), the coupling losses parameter is set to $\eta_{\mathrm{ext}}^{1}=0.13$. This low numerical value gives rise to small fluctuations around a $10 \%$ in the master laser output. As a consequence, almost no fluctuations of the peak powers of the output pulses is observed. On the other hand, the numerical value of $\eta_{\text {ext }}^{1}$ in Fig. 4(c) and (d) has been raised to unity. This situation would correspond to a grown structure in which no preferred propagation direction has been selected, such as a passive waveguide. In this case, large fluctuations in the $\mathrm{DFB}_{1}$ operation around a $60 \%$ are observed, which clearly lead to fluctuations of the peak powers of the optical pulses. However, we note that even in this unpleasant situation, the output pulses remain limited to their $50 \mathrm{ps}$ time slot in spite of their peak-power dispersion, and are thus suitable for multiplexing applications. 
Information regarding the frequency response of the device is supplied in Fig. 5. The optical spectrum [Fig. 5(b)] is clearly more symmetric than that of the solitary slave $\mathrm{DFB}_{2}$, which presents the typical downchirp of gain-switched semiconductor lasers [Fig. 5(a)]. The FWHM of the optical spectrum generated with our device is only slightly larger than that of solitary gain-switched lasers, mainly due to their narrow pulse shape.

In the reported results, the time delays in the modulator delay lines have been selected according to the following strategy. The gain of the free-running slave laser $\mathrm{DFB}_{2}$, provided by the carrier population, reaches its maximum just before the laser turns on, and then has a fast decay due to stimulated recombinations. This laser turn-on is usually triggered by spontaneous emission events. In our device the delay applied to $\mathrm{EAM}_{1}$ is selected to supply optical injection just before the slave gain reaches its maximum, avoiding a noiseinduced turn-on. The delay applied to $\mathrm{EAM}_{2}$ is determined afterwards so as to suppress secondary pulses due to the slave laser dynamics.

Although identical lasers have been used in the reported simulations, different master and slave lasers can be used, as long as the two lasers are single-mode and operate at the same optical frequency. Slight detunings of up to $100 \mathrm{MHz}$ do not lead to detrimental performances. In the case that two DFB lasers cannot match the frequency in such a way, multisection DBR lasers could be also used. The modulation bandwith of the master laser is not relevant, since it is $\mathrm{CW}$ operated. The slave laser and the two EAM's must allow a modulation bandwith of $5 \mathrm{GHz}$, a modulation rate commercialy available at present for these devices.

\section{CONCLUSIONS}

A low-cost generator of optical pulses based on modified injection-locking techniques has been demonstrated. The device incorporates two semiconductor lasers, one of them stabilized at a constant bias and the other current modulated according to a RZ periodic or pseudorandom bit stream. Width and height of the generated pulses are selected by means of controlled delays in the current applied to two electroabsorption modulators. These delays must be set so that the optical injection is supplied to the slave at the maximum of the material gain. In a particular configuration, a modulation bandwidth of $20 \mathrm{GHz}$ has been reported by time multiplexing four optical pulses in a single time slot. The quality of the generated pulses is better than those generated in previously reported configurations. Finally, the proposed device can be implemented in a small package of a few millimeters due to the reduced size of the individual components.

\section{REFERENCES}

[1] S. E. Miller and A. G. Chynoweth, Optical Fiber Telecommunications. New York: Academic, 1979.

[2] J. Gowar, Optical Communication Systems. Englewood Cliffs, NJ: Prentice-Hall, 1984.

[3] S. Sasaki, M. M. Choy, and N. K. Cheung, "Effects of dynamic spectral behavior and mode-partitioning of $1550 \mu \mathrm{m}$ distributed feedback lasers on Gbit/s transmission systems," Electron. Lett., vol. 24, pp. 26-28, 1988.
[4] G. P. Agrawal, Fiber-Optic Communication Systems. New York: Wiley, 1992

[5] G. P. Agrawal and N. K. Dutta, Long Wavelength Semiconductor Lasers. New York: Van Nostrand Reinhold, 1986.

[6] K. Petermann, Laser Diode Modulation and Noise. Dordrecht, The Netherlands: Kluwer, 1988.

[7] T. Shen, "Timing jitter in semiconductor lasers under pseudorandom word modulation," IEEE J. Lightwave Technol., vol. 7, pp. 1394-1399, 1989.

[8] C. R. Mirasso, P. Colet, and M. San Miguel, "Pulse statistics in singlemode semiconductor lasers modulated at gigahertz rates," Opt. Lett., vol. 16 , pp. 1753-1755, 1991.

[9] P. Colet, C. R. Mirasso, and M. San Miguel, "Memory diagram of single-mode semiconductor lasers," IEEE J. Quantum Electron., vol. 29, pp. 1624-1630, 1993.

[10] C. R. Mirasso, P. Colet, and M. San Miguel, "Dependence of timing jitter on bias level for single-mode semiconductor lasers under highspeed operation," IEEE J. Quantum Electron., vol. QE-29, pp. 23-32, 1993.

[11] _ "Pseudorandom word modulation of single-mode semiconductor lasers at GHz rates," Proc. Inst. Elect. Eng.-J (Optoelectronics), vol. 140, pp. 26-29, 1993.

[12] C. R. Mirasso, A. Valle, L. Pesquera, and P. Colet, "Simple method for estimating the memory diagram in single mode semiconductor lasers," Proc. Inst. Elect. Eng.-J (Optoelectronics), vol. 141, pp. 109-113, 1994.

[13] N. Holonyak, R. M. Kolbas, R. D. Dupuis, and P. D. Dapkus, "Quantumwell heterostructure lasers," IEEE J. Quantum Electron., vol. QE-16, pp. 170-186, 1980

[14] Y. Sasai, J. Ohya, M. Ogura, and T. Kajiwara, "Dynamic spectral linewidth in InGaAsP multiquantum well lasers grown by liquid-phase epitaxy," Electron. Lett., vol. 5, pp. 232-233, 1987.

[15] Y. Sasai, J. Ohya, and M. Ogura, "Spectral linewidth and resonantfrequency characteristics of InGaAsP/InP multiquantum well lasers," IEEE J. Quantum Electron., vol. QE-25, pp. 662-667, 1989.

[16] A. A. Tager and K. Petermann, "High-frequency oscillations and selfmode locking in short external-cavity laser-diodes," IEEE J. Quantum Electron., vol. QE-30, pp. 1553-1561, 1994.

[17] J. Dellunde and M. C. Torrent, "Optoelectronic feedback stabilization of current modulated laser diodes," Appl. Phys. Lett., vol. 68, pp. 1601-1603, 1996.

[18] K. A. Williams, I. H. White, D. Burns, and W. Sibbett, "Low jitter $Q$ switching using optoelectronic feedback," Electron. Lett., vol. 30, pp. 1687-1688, 1994.

[19] M. Schell, D. Huhse, A. G. Weber, G. Fischbeck, D. Bimberg, D. S. Tarasov, A. V. Gorbachov, and D. Z. Garbuzov, "20 nm wavelength tunable singlemode picosecond pulse generation at $1.3 \mu \mathrm{m}$ by selfseeded gain-switched semiconductor laser," Electron. Lett., vol. 28, pp. 2154-2155, 1992.

[20] D. Huhse, M. Schell, J. Kaessner, D. Bimberg, I. S. Tarasov, A. V. Gorbachov, and D. Z. Garbuzov, "Generation of electrically wavelength tunable $(\Delta \lambda=40 \mathrm{~nm})$ singlemode laser pulses from a 1.3 $\mu \mathrm{m}$ Fabry-Perot laser by self-seeding in a fiber-optic configuration," Electron. Lett., vol. 30, pp. 157-158, 1994.

[21] D. Huhse, M. Schell, W. Utz, J. Kaessner, and D. Bimberg, "Dynamics of single-mode formation in self-seeded Fabry-Perot laser diodes," IEEE Photon. Technol. Lett., vol. 7, pp. 351-353, 1995.

[22] L. N. Langley and K. A. Shore, "The effect of external optical feedback on the turn-on delay statistics of laser diodes under pseudorandom modulation," IEEE Photon. Technol. Lett., vol. 4, pp. 1207-1209, 1992.

[23] J. Dellunde, M. C. Torrent, C. R. Mirasso, and J. M. Sancho, "Turn-on time statistics of modulated lasers subjected to resonant weak optical feedback," Phys. Rev. A, vol. 52, pp. 4187-4193, 1995.

[24] J. Dellunde and A. Sapia, "Gain-switching of laser diodes coupled to optical fibers," IEEE Photon. Technol. Lett., vol. 7, pp. 1258-1260, 1995.

[25] K. Petermann, "External optical feedback phenomena in semiconductor lasers," IEEE J. Select. Topics Quantum Electron., vol. 2, pp. 480-489, 1995.

[26] S. Mohrdiek, H. Burkhard, and H. Walter, "Multigigabit/s standard fiber transmission by injection-locked directly modulated lasers," in Proc. COST Workshop'94 Optical Telecommunications, pp. 91-94.

[27] _ "Chirp reduction of directly modulated-lasers at $10 \mathrm{~Gb} / \mathrm{s}$ by strong CW light injection," J. Lightwave Technol., vol. LT-12, pp. 418-424, 1994.

[28] J. Dellunde, M. C. Torrent, J. M. Sancho, and M. San Miguel, "Gainswitching and frequency chirp of injection-locked single-mode semi- 
conductor lasers," in Tech. Dig. Nonlinear Dynamics Optical Systems Conference NDOS, 1995.

[29] R. Lang, "Injection locking properties of a semiconductor-laser," IEEE J. Quantum Electron., vol. QE-18, pp. 976-983, 1982.

[30] F. Mogensen, H. Olesen, and G. Jacobsen, "Locking conditions and stability properties for a semiconductor laser with external light injection," IEEE J. Quantum Electron., vol. QE-21, pp. 784-793, 1985.

[31] I. Petitbon, P. Gallion, G. Debarge, and C. Chabran, "Locking bandwidth and relaxation oscillations of an injection-locked semiconductor laser," IEEE J. Quantum Electron., vol. 24, pp. 148-154, 1988.

[32] M. C. Torrent, S. Balle, M. San Miguel, and J. M. Sancho, "Detection of a weak external signal via the switch-on time statistics of a semiconductor laser," Phys. Rev. A, vol. 47, pp. 3390-3395, 1993.

[33] D. S. Seo, H. F. Liu, D. Y. Kim, and D. Sampson, "Injection power and wavelength dependence of an external-seeded gain-switched Fabry-Perot laser," Appl. Phys. Lett., vol. 67, pp. 1503-1505, 1995.

[34] D. S. Seo, D. Y. Kim, and H. F. Liu, "Timing jitter reduction of gainswitched DFB laser by external injection-seeding," Electron. Lett., vol. 32, pp. 44-45, 1996.

[35] C. Lin, J. K. Andersen, and F. Mengel, "Frequency chirp reduction in a $2.2 \mathrm{Gbit} / \mathrm{s}$ directly modulated InGaAsP semiconductor laser by $\mathrm{CW}$ injection," Electron. Lett., vol. 21, pp. 80-81, 1985.

[36] Optical Fiber Telecommunications II, S. E. Miller and I. P. Kaminow, Eds. Academic, 1988.

[37] C. R. Mirasso, "Pulse width reduction in single mode diode lasers via external injection of optical pulses," Appl. Phys. Lett., vol. 66, pp. $1880-1882,1995$.

[38] S. Balle, P. Colet, and M. San Miguel, "Statistics for the transient response of single-mode semiconductor laser gain switching," Phys. Rev. A, vol. 43, pp. 498-506, 1991.

[39] S. Balle, N. B. Abraham, P. Colet, and M. San Miguel, "Parametric dependence of stochastic frequency variations in gain switching of a single-mode laser diode," IEEE J. Quantum Electron., vol. QE-29, pp. 33-41, 1993

[40] A. Sapia, P. Spano, C. R. Mirasso, P. Colet, and M. San Miguel, "Pattern effects in time jitter of semiconductor lasers," Appl. Phys. Lett., vol. 61 , pp. 1748-1750, 1991.

[41] G. P. Agrawal and G. R. Gray, "Effect of phase-conjugate feedback on the noise characteristics of semiconductor lasers," Phys. Rev. A, vol. 46, pp. 5890-5898, 1992.

[42] M. Schell, D. Huhse, W. Utz, J. Kässner, D. Bimberg, and D. S. Tarasov, "Jitter and dynamics of self-seeded Fabry-Perot laser diodes," IEEE J. Select. Topics Quantum Electron., vol. 2, pp. 528-534, 1995

[43] D. Marcuse and T. H. Wood, "Simulation of a laser modulator driven by NRZ pulses," J. Lightwave Technol., vol. 14, pp. 860-866, 1996.

[44] K. Wakita, K. Sato, I. Kotaka, M. Yamamoto, and M. Asobe, "Transform-limited 7-ps optical pulse generation using a sinusoidally driven InGaAsP/InGaAsP strained multi-quantum-well DFB lasers/modulator monolithically integrated light sources," IEEE Photon. Technol. Lett., vol. 5, pp. 899-901, 1993.

[45] R. Lang and K. Kobayashi, "External optical feedback effects on semiconductor injection laser properties," IEEE J. Quantum Electron., vol. QE-16, pp. 347-355, 1980
[46] C. R. Mirasso, J. M. Sancho, J. Dellunde, and M. C. Torrent, "Low-cost feedback-insensitive DFB laser/electroabsorption modulator package," IEEE Photon. Technol. Lett., vol. 9, pp. 1000-1001, 1997.

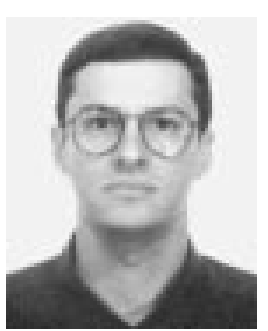

Jaume Dellunde was born in Barcelona, Spain, on May 9, 1968. He received the M.S. and Ph.D. degrees from the Universitat de Barcelona, Spain, in 1991 and 1996, respectively. The subject of the $\mathrm{Ph}$.D. degree dissertation was the influence of optical injection and optical feedback on laser diodes His current interests include new semiconductor laser structures and photonic switches.

C. R. Mirasso, photograph and biography not available at the time of publication.

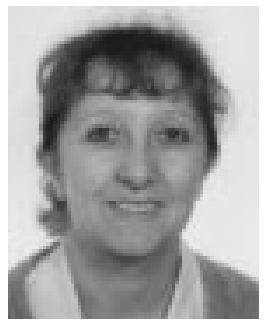

M. C. Torrent received the B.S., M.S., and Ph.D degrees in physics from the University of Barcelona, Spain.

In 1991, she joined the Department of Physics and Nuclear Engineering, Politechnical University of Catalonia, Spain, as a member of the faculty. Her scientific interests have gradually changed from theoretical problems in statistical mechanics to applied physics. Her current research is on spatio-temporal dynamics of broad-area semiconductor and threelevel lasers and nonlinear dynamics of semiconductor and microchip laser arrays.

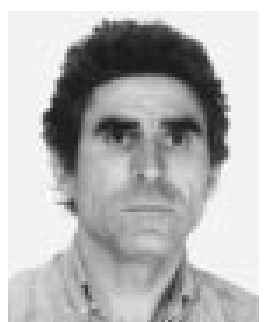

Jose M. Sancho received the Ph.D. degree in physics in 1980 from the University of Barcelona, Spain.

Since 1987, he has been a Full Professor of Condensed Matter, University of Barcelona. He is presently a Visiting Scholar at the Institute of Nonlinear Science, University of California at San Diego, La Jolla. 